\title{
The AGL equation from the dipole picture
}

\author{
M.B. Gay Ducati ${ }^{1}$, V.P. Gonçalves ${ }^{2}$ \\ Instituto de Física, Univ. Federal do Rio Grande do Sul, Caixa Postal 15051, \\ 91501-970 Porto Alegre, RS, Brazil
}

Received 23 March 1999; revised 12 May 1999; accepted 7 June 1999

\begin{abstract}
The AGL equation includes all multiple pomeron exchanges in the double logarithmic approximation (DLA) limit, leading to a unitarized gluon distribution in the small $x$ regime. This equation was originally obtained using the Glauber-Mueller approach. We demonstrate in this paper that the AGL equation and, consequently, the GLR equation, can also be obtained from the dipole picture in the double logarithmic limit, using an evolution equation, recently proposed, which includes all multiple pomeron exchanges in the leading logarithmic approximation. Our conclusion is that the AGL equation is a good candidate for a unitarized evolution equation at small $x$ in the DLA limit. (C) 1999 Elsevier Science B.V. All rights reserved.
\end{abstract}

PACS: 11.80.La; 24.95.+p;

Keywords: Small-x QCD; Unitarity corrections; Evolution equation

\section{Introduction}

The physics of high-density QCD has become an increasingly active subject of research, both from experimental and theoretical points of view. Presently, and in the near future, the collider facilities such as the DESY ep collider HERA $(e p, e A)$, Fermilab Tevatron $(p \bar{p}, p A)$, BNL Relativistic Heavy Ion Collider (RHIC), and CERN Large Hadron Collider (LHC) ( $p \bar{p}, A A$ ) will be able to probe new regimes of dense quark matter at very small Bjorken $x$ or/and at large $A$, with rather different dynamical properties. The description of these processes is directly associated with a correct description of the dynamics in this kinematical region.

\footnotetext{
${ }^{1}$ E-mail:gay@if.ufrgs.br

${ }^{2}$ E-mail:barros@if.ufrgs.br
} 
The behavior of the cross sections in the high-energy limit $(s \rightarrow \infty)$ and fixed momentum transfer is expected to be described by the BFKL equation [1]. The simplest process where this equation applies is the high-energy scattering between two heavy quark-antiquark states, i.e. the onium-onium scattering. For a sufficiently heavy onium state, high-energy scattering is a perturbative process since the onium radius gives the essential scale at which the running coupling $\alpha_{s}$ is evaluated. This process was studied in the dipole picture [2-4], where the heavy quark-antiquark pair and the soft gluons in the limit of large number of colors $N_{c}$ are viewed as a collection of color dipoles. In this case, the cross section can be understood as a product of the number of dipoles in one onium state times the number of dipoles in the other onium state times the basic cross section for dipole-dipole scattering due to two-gluon exchange. The cross section grows rapidly with the energy $\left(\sigma \propto \alpha_{s}^{2} e^{\left(\alpha_{P}-1\right) Y}\right.$, where $\left(\alpha_{P}-1\right)=\frac{1}{\pi}\left(4 \alpha_{s} N_{c}\right) \ln 2$ and $Y=\ln s / Q^{2}$ ) because the number of dipoles in the light-cone wavefunction grows rapidly with the energy. In Ref. [2] Mueller demonstrated that the QCD dipole picture reproduces the BFKL physics.

One of the main characteristics of the BFKL equation is that it predicts a very high density of partons in the small $x$ region. Therefore a new dynamical effect associated with the unitarity corrections is expected to stop further growth of the parton densities. The understanding of the unitarity corrections has been a challenge of perturbative QCD (PQCD).

About seventeen years ago, Gribov, Levin, and Ryskin (GLR) [5] performed a detailed study of this problem in the double logarithmic approximation (DLA). They argued that the physical processes of interaction and recombination of partons become important in the parton cascade at a large value of the parton density, and that these shadowing corrections could be expressed in a new evolution equation - the GLR equation. This equation considers the leading non-ladder contributions: the multi-ladder diagrams, denoted as fan diagrams. The main characteristics of this equation are that it predicts a saturation of the gluon distribution at very small $x$, it predicts a critical line, separating the perturbative regime from the saturation regime, and it is only valid in the border of this critical line. Therefore, the GLR equation predicts the limit of its validity. In the last decade, the solution [6-8] and possible generalizations [9,10] of the GLR equation have been studied in great detail. Recently, an eikonal approach to the shadowing corrections was proposed in the literature [11-13]. The starting point of these papers is the proof of the Glauber formula in QCD [14], which considers only the interaction of the fastest partons with the target. In Ref. [12], a generalized equation which takes into account the interaction of all partons in a parton cascade with the target in the DLA limit was proposed by Ayala, Gay Ducati, and Levin (AGL). The main properties of the generalized equation are that (i) the iterations of this equation coincide with the iteration of the Glauber-Mueller formula; (ii) its solution matches the solution of the DGLAP evolution equation in the DLA limit of PQCD; (iii) it has the GLR equation as a limit, and (iv) it contains the Glauber-Mueller formula. Therefore, the AGL equation is valid in a large kinematical region. 
The AGL equation resums all multiple pomeron exchanges in the DLA limit. Its asymptotic solution is given by $x G \propto Q^{2} R^{2} \ln (1 / x)$, where $R$ is the size of the target, i.e. differently from the GLR equation, it does not predict saturation of the gluon distribution in the very small $x$ limit.

The unitarity corrections in the leading logarithmic limit can be studied using the dipole picture. In this picture the unitarity corrections (multiple interactions between the onia) become important at high energies $[15,16]$. These corrections in general are neglected as being of higher order in $1 / N_{c}$. However, when the energy is high, there is a large number of dipoles in each onium, and the total number of possible interactions is of order $e^{\left(\alpha_{P}-1\right)(y+(Y-y))}$ (the product of the number of dipoles in each onium) [16]. So when $\alpha_{s}^{2} e^{\left(\alpha_{P}-1\right) Y} \approx 1$, one should take into account the multiple scattering corrections - multiple pomeron exchange - despite the fact that they are suppressed by $1 / N_{c}^{2}$. Recently, an equation which includes all pomeron exchanges in the leading logarithmic approximation using the dipole picture was proposed (Eq. (15) in Ref. [17]). In this paper we will denote this equation as $K$ equation. As the $K$ equation and the AGL equation resums the same group of diagrams, both should be identical in a common limit. Our goal in this paper is to demonstrate that the $\mathrm{K}$ equation reproduces the AGL equation and, consequently, the GLR equation in the DLA limit.

This paper is organized as follows. In Section 2 the AGL approach for the unitarity corrections is briefly reviewed. We clarify some steps and obtain the GLR equation as a limit case. In Section 3 we obtain the nuclear structure function $F_{2}^{A}$ in the GlauberMueller approach and compare it with the expression proposed in [17]. It allows us to obtain precisely the relation between the propagator of the $q \bar{q}$ through the nucleus and the gluon distribution. In Section 4, we obtain the AGL equation from the dipole picture and, as a limit, the GLR equation. Finally, in Section 5, we present our conclusions.

\section{The AGL equation}

In the nucleus rest frame we can consider the interaction between a virtual colorless hard probe and the nucleus via a gluon pair $(g g)$ component of the virtual probe. In the region where $x \ll 1 / 2 m R$ ( $R$ is the size of the target), the $g g$ pair crosses the target with fixed transverse distance $r_{t}$ between the gluons. Moreover, at high energies the lifetime of the $g g$ pair may substantially exceed the nuclear radius. The cross section for this process is written as [11]

$$
\sigma^{G^{*} A}=\int_{0}^{1} d z \int \frac{d^{2} r_{t}}{\pi}\left|\Psi_{t}^{G^{*}}\left(Q^{2}, r_{t}, x, z\right)\right|^{2} \sigma^{g g+A}\left(z, r_{t}^{2}\right),
$$

where $G^{*}$ is the virtual colorless hard probe with virtuality $Q^{2}, z$ is the fraction of energy carried by the gluon and $\Psi_{t}^{G^{*}}$ is the wave function of the transverse polarized gluon in the virtual probe. Furthermore, $\sigma^{g g+A}\left(z, r_{t}^{2}\right)$ is the cross section of the interaction of the $g g$ pair with the nucleus. 
As our goal in this paper is to obtain the AGL from the dipole picture, we should make a transformation of Eq. (1) for $q \bar{q}$ dipoles, since in this picture they are the basic configuration. Considering that $\sigma^{g g+A}=\left(C_{A} / C_{F}\right) \sigma^{q \bar{q}}$ we have that the cross section for the interaction between the virtual probe $G^{*}$ and the nucleus via the $q \bar{q}$ component of the virtual probe is given by

$$
\sigma^{G^{*} A}=\frac{C_{A}}{C_{F}} \int_{0}^{1} d z \int \frac{d^{2} r_{t}}{\pi}\left|\Psi_{t}^{G^{*}}\left(Q^{2}, r_{t}, x, z\right)\right|^{2} \sigma^{q \bar{q}+A}\left(z, r_{t}^{2}\right)
$$

To estimate the unitarity corrections we have to take into account the rescatterings of the quark-antiquark pair inside the nucleus. The contributions of the rescatterings can be estimated using the Glauber-Mueller approach proposed in Ref. [11]. Following the same steps used in [11], i.e. considering the $s$-channel unitarity and the eikonal model, one obtains that the $\sigma^{G^{*} A}$ cross section is written as

$$
\sigma^{G^{*} A}=\frac{C_{A}}{C_{F}} \int_{0}^{1} d z \int \frac{d^{2} r_{t}}{\pi} \int \frac{d^{2} b_{t}}{\pi}\left|\Psi_{t}^{G^{*}}\left(Q^{2}, r_{t}, z\right)\right|^{2} 2\left[1-e^{-\frac{1}{2} \sigma_{N}^{q \bar{q}}\left(x^{\prime}, 4 / r_{t}^{2}\right) S\left(b_{t}\right)}\right],
$$

where $x^{\prime}=x /\left(z r_{t}^{2} Q^{2}\right.$ ) ( $x$ is the Bjorken variable), $b_{t}$ is the impact parameter, $S\left(b_{t}\right)=$ $\left(A / \pi R^{2}\right) e^{-b_{t}^{2} / R^{2}}$ is the gaussian profile function and $\sigma_{N}^{q \bar{q}}$ is the cross section of the interaction of the $q \bar{q}$ pair with the nucleons inside the nucleus. In Ref. [18] the authors have shown that $\sigma_{N}^{q \bar{q}}=\frac{C_{F}}{C_{A}}\left(3 \alpha_{s}\left(4 / r_{t}^{2}\right) / 4\right) \pi^{2} r_{t}^{2} x G_{N}\left(x, 4 / r_{t}^{2}\right)$, where $x G_{N}\left(x, 4 / r_{t}^{2}\right)$ is the nucleon gluon distribution.

The wavefunction $\Psi^{G^{*}}$ was calculated in [14,11] using the technique of Ref. [19]. Here we only explicitate the result for the squared wavefunction, which is given by

$$
\left|\Psi_{t}^{G^{*}}\left(Q^{2}, r_{t}, z\right)\right|^{2}=\frac{1}{z(1-z)}\left[\left(\epsilon^{2} K_{2}\left(\epsilon r_{t}\right)-\frac{\epsilon K_{1}\left(\epsilon r_{t}\right)}{r_{t}}\right)^{2}+\frac{1}{r_{t}^{2}}\left(\epsilon K_{\mathrm{I}}\left(\epsilon r_{t}\right)\right)^{2}\right] \text {, }
$$

where $\epsilon^{2}=Q^{2} z(1-z)$ and the $K_{i}$ are the modified Bessel functions. The main contribution in expression (3) comes from the region of small $z$, where $\epsilon r_{t} \ll 1$. Using the expansion of the modified Bessel functions for small values of the argument gives that the squared wavefunction simplifies to

$$
\left|\Psi_{t}^{G^{*}}\left(Q^{2}, r_{t}, x, z\right)\right|^{2}=\frac{2}{z r_{t}^{4}} .
$$

The condition $\epsilon r_{t} \ll 1$ implies that

$$
z(1-z)<\frac{1}{Q^{2} r_{t}^{2}}<\frac{1}{4}
$$

where the last inequality comes from symmetric pairs $(z=1 / 2)$. From the expression (6) results that $x^{\prime} \geqslant x$. 


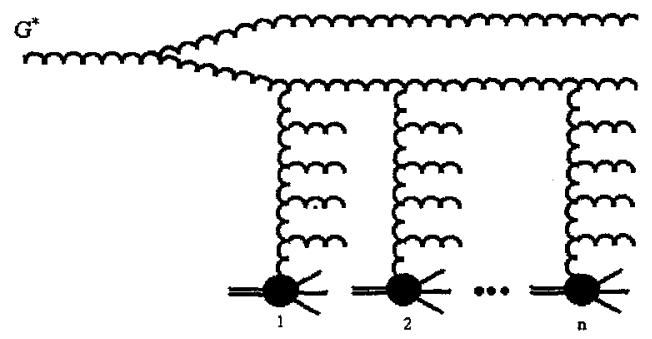

Fig. 1. Space-time picture of the Glauber-Mueller approach. The virtual colorless hard probe $G^{*}$ decays into a pair with transverse distance $r_{t}$ which interacts with the $n$ nucleons within the nucleus.

The relation $\sigma^{G^{*} A}\left(x, Q^{2}\right)=\left(4 \pi^{2} \alpha_{s} / Q^{2}\right) x G_{A}\left(x, Q^{2}\right)$ is valid for a virtual probe $G^{*}$ with virtuality $Q^{2}$. Consequently, using the expression (5) of the squared wavefunction and making the change of variables $z \rightarrow x^{\prime}$, we obtain that the Glauber-Mueller formula for the interaction of the $q \bar{q}$ pair with the nucleus is written as

$$
x G_{A}\left(x, Q^{2}\right)=\frac{4}{\pi^{2}} \frac{C_{A}}{C_{F}} \int_{x}^{1} \frac{d x^{\prime}}{x^{\prime}} \int_{4 / Q^{2}}^{\infty} \frac{d^{2} r_{t}}{\pi r_{t}^{4}} \int \frac{d^{2} b_{t}}{\pi} 2\left[1-e^{-\frac{1}{2} \sigma_{N}^{\bar{q}}\left(x^{\prime}, 4 / r_{t}^{2}\right) S\left(b_{t}\right)}\right] .
$$

The lower limit in $r_{t}$ integration comes from the expression (6).

The space-time picture of the Glauber-Mueller approach is presented in Fig. 1. It takes into account only the interaction of the fastest partons with the target. As in QCD we expect that all partons from the cascade interact with the target, a generalized equation was proposed in [11].

The AGL equation can be obtained directly from $\mathrm{Eq}$. (7) differentiating this formula with respect to $\ln 1 / x$ and $\ln Q^{2} / \Lambda_{\mathrm{QCD}}^{2}$. Therefore the AGL for the interaction of $q \bar{q}$ dipole is given by

$$
\frac{\partial^{2} x G_{A}\left(x, Q^{2}\right)}{\partial \ln (1 / x) \partial \ln \left(Q^{2} / \Lambda_{\mathrm{QCD}}^{2}\right)}=\frac{2 Q^{2}}{\pi^{2}} \frac{C_{A}}{C_{F}} \int \frac{d^{2} b_{t}}{\pi}\left[1-e^{-\frac{1}{2} \sigma_{N}^{q \bar{q}}\left(x, Q^{2}\right) S\left(b_{t}\right)}\right],
$$

where the dependence of $\sigma_{N}^{q \bar{q}}$ in the virtuality of the virtual probe results from the derivative. The non-perturbative effects coming from the large distances are absorbed in the boundary and initial conditions. This equation is valid in the double logarithmic approximation (DLA).

For a central collision $(b=0)$ the AGL equation (8) reduces to

$$
\frac{\partial^{2} x G_{A}\left(x, Q^{2}\right)}{\partial \ln (1 / x) \partial \ln \left(Q^{2} / \Lambda_{\mathrm{QCD}}^{2}\right)}=\frac{C_{A}}{C_{F}} \frac{2 Q^{2} R^{2}}{\pi^{2}}\left[1-e^{-\frac{1}{2} \sigma_{N}^{q \bar{q}}\left(x, Q^{2}\right) S(0)}\right],
$$

where we have set $d b_{t}^{2}=R^{2}$ for a $b=0$ collision. This limit is important since the cross section is strongly unitarized at small impact parameters $[12,16]$.

Considering that the transverse cross-sectional area of the nucleus is $S_{\perp}=\pi R^{2}$ and that $S(0)=A /\left(\pi R^{2}\right)$, the AGL equation for $b=0$ is obtained as 


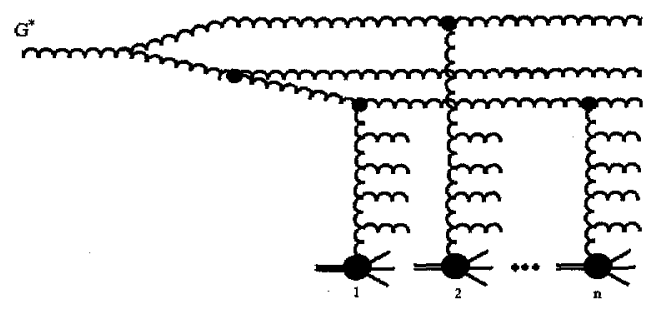

Fig. 2. First iteration of the Glauber-Mueller approach. The AGL equation takes into account the interation of all partons of the parton cascade with several nucleons within of the nucleus.

$$
\frac{\partial^{2} x G_{A}\left(x, Q^{2}\right)}{\partial \ln (1 / x) \partial \ln \left(Q^{2} / \Lambda_{\mathrm{QCD}}^{2}\right)}=\frac{N_{c} C_{F} S_{\perp}}{\pi^{3}} Q^{2}\left[1-e^{-\left(2 \alpha_{s} \pi^{2} / N_{c} S_{\perp}\right) \frac{1}{Q^{2}} x G_{A}\left(x, Q^{2}\right)}\right],
$$

where $N_{c}=3$ and we have assumed that $C_{F}=N_{c} / 2$ in the large $N_{c}$ limit. Eq. (10) agrees with the expression (2) of [17].

The AGL equation takes into account the interaction of all partons in a parton cascade with the target. In other words, the AGL equation takes into account that each parton in the parton cascade interacts with several nucleons within of the nucleus (Glauber multiple scattering). In Fig. 2 we present the result of the first iteration from the Glauber-Mueller formula, which is one of the terms summed by the AGL equation.

The GLR equation can be obtained directly from $\mathrm{Eq}$. (10). If we expand the righthand side of this equation to the second order in $x G_{A}$ we obtain

$$
\frac{\partial^{2} x G_{A}\left(x, Q^{2}\right)}{\partial \ln (1 / x) \partial \ln \left(Q^{2} / \Lambda_{\mathrm{QCD}}^{2}\right)}=\frac{\alpha_{s} N_{c}}{\pi} x G_{A}\left(x, Q^{2}\right)-\frac{\alpha_{s}^{2} \pi}{S_{\perp}} \frac{1}{Q^{2}}\left[x G_{A}\left(x, Q^{2}\right)\right]^{2},
$$

which is the GLR equation for a cylindrical nucleus case (see Eq. (19) in [17]). Moreover, if the unitarity corrections are small, only the first order in $x G_{A}$ contributes. In this limit, Eqs. (10) and (11) match with the DGLAP evolution equation in the DLA limit.

Therefore the AGL equation (i) matches the DGLAP evolution equation in the DLA limit of PQCD, (ii) it has the GLR equation as a limit, and (iii) contains the GlauberMueller formula. We have that the AGL equation is valid in a large kinematical region.

Recently, a comprehensive phenomenological analysis of the behavior of distinct observables was made for the HERA kinematical region using the Glauber-Mueller approach $[13,22,23]$. In this kinematical region the solutions from the AGL equation and the Glauber-Mueller approximately coincide. The results from these analysis agree with the recent HERA data and allows us to make some predictions which will be tested in the near future. In Ref. [22] we have analysed the behavior of the longitudinal structure function $F_{L}$ and the charm component of the proton structure function $F_{2}^{c}$ and have shown that our results agree with the $\mathrm{H} 1$ data. New data, with better statistics, will allow us to evidentiate the unitarity corrections. In Ref. [23] we have shown that the recent ZEUS data can be described if the unitarity corrections for the $F_{2}$ slope are considered. Our main conclusion is that the unitarity corrections cannot be disregarded in the HERA kinematical region. 


\section{The nuclear structure function}

The unitarity corrections to the nuclear structure function can be estimated in the rest frame of the target. This intuitive point of view was proposed by Gribov many years ago [24]. Gribov's assumption is that at small values of $x$ the virtual photon fluctuates into a $q \bar{q}$ pair well before the interaction with the target, and this system interacts with the target. This formalism has been established as a useful tool for calculating deep inelastic and related diffractive cross section for $\gamma^{*} p$ scattering in the last years $[25,26]$. The Gribov factorization follows from the fact that the lifetime of the $q \bar{q}$ fluctuation is much larger than the time of the partonic interactions. According to the uncertainty principle, the fluctuation time is $\approx 1 / m x$, where $m$ denotes the target mass.

The space-time picture of the DIS in the target rest frame can be viewed as the decay of the virtual photon at high energy $(\operatorname{small} x$ ) into a quark-antiquark pair long before the interaction with the target. The $q \bar{q}$ pair subsequently interacts with the target. In the small $x$ region, where $x \ll 1 / 2 m R$, the $q \bar{q}$ pair crosses the target with fixed transverse distance $r_{t}$ between the quarks. It allows us to factorize the total cross section between the wave function of the photon and the interaction cross section of the quark-antiquark pair with the target. The photon wave function is calculable and the interaction cross section is modelled. Therefore, the nuclear structure function is given by

$$
F_{2}^{A}\left(x, Q^{2}\right)=\frac{Q^{2}}{4 \pi \alpha_{\mathrm{em}}} \int d z \int \frac{d^{2} r_{t}}{\pi}\left|\Psi\left(z, r_{t}\right)\right|^{2} \sigma^{q \bar{q}+A}\left(z, r_{t}\right),
$$

where

$$
\left|\Psi\left(z, r_{t}\right)\right|^{2}=\frac{6 \alpha_{\mathrm{em}}}{(2 \pi)^{2}} \sum_{i}^{n_{f}} e_{f}^{2}\left\{\left[z^{2}+(1-z)^{2}\right] \epsilon^{2} K_{1}\left(\epsilon r_{t}\right)^{2}+m_{f}^{2} K_{0}^{2}\left(\epsilon r_{t}\right)^{2}\right\},
$$

$\alpha_{\mathrm{em}}$ is the electromagnetic coupling constant, $\epsilon=z(1-z) Q^{2}+m_{f}^{2}, m_{f}$ is the quark mass, $n_{f}$ is the number of active flavors, $e_{f}^{2}$ is the square of the parton charge (in units of $e$ ), $K_{0,1}$ are the modified Bessel functions and $z$ is the fraction of the photon's light-cone momentum carried by one of the quarks of the pair. In the leading $\log (1 / x)$ approximation we can neglect the change of $z$ during the interaction and describe the cross section $\sigma^{q \bar{q}+A}\left(z, r_{t}^{2}\right)$ as a function of the variable $x$.

We estimated the unitarity corrections considering the Glauber multiple scattering theory [27], which was probed in QCD [14]. The nuclear collision is analysed as a succession of collisions of the probe with individual nucleons within the nucleus, which implies that the $F_{2}$ structure function can be written as [11]

$$
F_{2}^{A}\left(x, Q^{2}\right)=\frac{Q^{2}}{4 \pi \alpha_{\mathrm{em}}} \int d z \int \frac{d^{2} r_{t}}{\pi}\left|\Psi\left(z, r_{t}\right)\right|^{2} \int \frac{d^{2} b_{t}}{\pi} 2\left\{1-e^{-\frac{1}{2} \Omega_{\bar{q} \bar{q}}\left(x, r_{t}, b_{t}\right)}\right\},
$$

where the opacity $\Omega_{q \bar{q}}\left(x, r_{t}, b_{t}\right)$ describes the interaction of the $q \bar{q}$ pair with the target.

In the region where $\Omega_{q \bar{q}}$ is small $\left(\Omega_{q \bar{q}} \ll 1\right)$ the $b_{t}$ dependence can be factorized as $\Omega_{q \bar{q}}=\overline{\Omega_{q \bar{q}}} S\left(b_{t}\right)$ [5], with the normalization $\int d^{2} b_{t} S\left(b_{t}\right)=1$. The eikonal approach assumes that the factorization of the $b_{t}$ dependence $\Omega_{q \bar{q}}=\overline{\Omega_{q \bar{q}}} S\left(b_{t}\right)$, which is valid in 
the region where $\Omega_{q \bar{q}}$ is small, occurs in the whole kinematical region [12]. The main assumption of the eikonal approach in pQCD is the identification of opacity $\overline{\Omega_{q \bar{q}}}$ with the gluon distribution. In Ref. [18] the opacity is given by $\overline{\Omega_{q \bar{q}}}=\sigma_{N}^{q \bar{q}}$.

For a central collision $(b=0), \mathrm{Eq}$. (14) reduces to

$$
F_{2}^{A}\left(x, Q^{2}\right)=\frac{Q^{2}}{4 \pi \alpha_{\mathrm{em}}} R^{2} \int d z \int \frac{d^{2} r_{t}}{\pi}\left|\Psi\left(z, r_{t}\right)\right|^{2} 2\left\{1-e^{-\frac{1}{2} \sigma_{N}^{q \bar{q}} S(0)}\right\} .
$$

Substituting $\sigma_{N}^{q \bar{q}}$ and $S(0)$ we obtain that the nuclear structure function for $b=0$ is given by

$$
F_{2}^{A}\left(x, Q^{2}\right)=\frac{Q^{2}}{4 \pi \alpha_{\mathrm{em}}} R^{2} \int d z \int \frac{d^{2} r_{t}}{\pi}\left|\Psi\left(z, r_{t}\right)\right|^{2} 2\left\{1-e^{-\left(\alpha_{s} C_{F} \pi^{2} / N_{c}^{2} S_{\perp}\right) r_{t}^{2} A x G\left(x, 1 / r_{t}^{2}\right)}\right\} .
$$

This expression estimates the unitarity corrections to the nuclear structure function for central collisions $(b=0)$ in the DLA limit using the Glauber-Mueller approach.

Comparing Eq. (16) with the expression to the nuclear structure function in the dipole picture proposed in [17], we obtain the representation for the total cross section of the $q \bar{q}$ pair interacting with the nucleus, $N\left(\boldsymbol{x}_{01}, \boldsymbol{b}_{0}=0, Y\right)$, used in that reference,

$$
N\left(\boldsymbol{x}_{01}, \boldsymbol{b}_{0}=0, Y\right)=2\left\{1-e^{-\left(\alpha_{s} C_{F} \pi^{2} / N_{c}^{2} S_{\perp}\right) x_{01}^{2} A x G\left(x, 1 / x_{01}^{2}\right)}\right\},
$$

where $x_{01}=x_{0}-x_{1} \equiv r_{t}\left[x_{0}\left(x_{1}\right)\right.$ is the transverse coordinate of the quark (antiquark) $]$ and $Y=\ln \left(s / Q^{2}\right)=\ln (1 / x)$. This relation between $N\left(x_{01}, b_{0}=0, Y\right)$ and the gluon distribution is valid in the DLA limit and is two times the propagator of the $q \bar{q}$ pair through the nucleus obtained in [20] at large $Q^{2}$ (see Eq. (6b) of Ref. [17]).

We will assume that the correct expression for $N$ in the DLA limit is given by (17). In the next section we will use this expression as an input in the evolution equation for $N$ obtained in the dipole picture.

\section{The AGL equation from the dipole picture}

Considering the multiple pomeron exchange, Kovchegov [17] has obtained an evolution equation for $N\left(\boldsymbol{x}_{01}, \boldsymbol{b}_{0}, Y\right)$ in the leading logarithmic approximation,

$$
\begin{aligned}
N\left(x_{01}, b_{0}, Y\right)= & -\gamma\left(x_{01}, b_{0}\right) \exp \left[-\frac{4 \alpha_{s} C_{F}}{\pi} \ln \left(\frac{x_{01}}{\rho}\right) Y\right] \\
& +\frac{\alpha_{s} C_{F}}{\pi^{2}} \int_{0}^{Y} d y \exp \left[-\frac{4 \alpha_{s} C_{F}}{\pi} \ln \left(\frac{x_{01}}{\rho}\right)(Y-y)\right] \\
& \times \int_{\rho} d^{2} x_{2} \frac{x_{01}^{2}}{x_{02}^{2} x_{12}^{2}}\left[2 N\left(x_{02}, b_{0}, y\right)-N\left(x_{02}, b_{0}, y\right) N\left(x_{12}, b_{0}, y\right)\right],
\end{aligned}
$$


where $x_{i j}=x_{i}-x_{j}$ is the size of the dipole with a quark in the transverse coordinate $x_{i}$ and the antiquark in $x_{j}, \gamma\left(x_{01}, b_{0}\right)$ is the propagator of the $q \bar{q}$ pair through the nucleus and $\rho$ is an ultraviolet cutoff in the equation which disappears in the physical quantities. We have reobtained this equation and denote it as the $\mathrm{K}$ equation.

Eq. (18) was obtained considering the scattering of a virtual photon with a nucleus. The physical picture for this interaction is the same as the Glauber-Mueller approach. The incoming virtual photon generates a $q \bar{q}$ pair which develops a cascade of gluons, which then scatters on the nucleus. In the large $N_{c}$ limit the gluon can be represented as a $q \bar{q}$ pair. Therefore, in this limit and in the leading logarithmic approximation, the cascade of gluons can be interpreted as a dipole cascade, where each dipole in the cascade interacts with several nucleons within the nucleus. Therefore, as the $\mathrm{K}$ equation and the AGL equation, although with distinct basic objects, resums the multiple scatterings of its respective degree of freedom, we expect that both coincide in a common limit.

In the double logarithmic limit, where the momentum scale of the photon $Q^{2}$ is larger than the momentum scale of the nucleus $\Lambda_{\mathrm{QCD}}$, we take the large $Q^{2}$ limit from (18), which reduces to

$$
\begin{aligned}
\frac{\partial N\left(\boldsymbol{x}_{01}, \boldsymbol{b}_{0}, Y\right)}{\partial Y}= & \frac{\alpha_{s} C_{F}}{\pi} x_{01}^{2} \int_{x_{01}^{2}}^{1 / \Lambda_{\mathrm{OCD}}^{2}} \frac{d x_{02}^{2}}{\left(x_{02}^{2}\right)^{2}}\left[2 N\left(\boldsymbol{x}_{02}, \boldsymbol{b}_{0}, Y\right)\right. \\
& \left.-N\left(\boldsymbol{x}_{02}, \boldsymbol{b}_{0}, Y\right) N\left(\boldsymbol{x}_{02}, \boldsymbol{b}_{0}, Y\right)\right]
\end{aligned}
$$

This equation describes the evolution of the dipole transverse sizes from a small scale $x_{01}$ to a large scale $1 / \Lambda_{\mathrm{QCD}}$. Differentiating the above expression with respect to $\ln \left(1 / x_{01}^{2} \Lambda_{\mathrm{QCD}}^{2}\right)$ yields a double differential equation for $N\left(x_{01}, b_{0}, Y\right)$,

$$
\frac{\partial^{2} N\left(\boldsymbol{x}_{01}, \boldsymbol{b}_{0}, Y\right)}{\partial Y \partial \ln \left(1 / x_{01}^{2} \Lambda_{\mathrm{QCD}}^{2}\right)}=\frac{\alpha_{s} C_{F}}{\pi}\left[2-N\left(\boldsymbol{x}_{01}, \boldsymbol{b}_{0}, Y\right)\right] N\left(\boldsymbol{x}_{01}, \boldsymbol{b}_{0}, Y\right)
$$

The physical picture for the dipole evolution in the DLA limit is that the produced dipoles at each step of the evolution have much greater transverse dimensions than the parent dipoles.

In Ref. [17] the connection between the quantity $N\left(x_{01}, b_{0}, Y\right)$ and the nuclear gluon distribution was discussed. As there is some freedom in the definition of the gluon distribution, the choice for the connection between the two functions was arbitrary. Here we use the result obtained in the previous section (Eq. (17)) to make this connection more explicit. If Eq. (17) is expanded to the first order in $x G_{A}$, our result differs from the choice used in [17] by a factor of 2 .

Substituting Eq. (17) into Eq. (20) and using $x_{01} \approx 2 / Q[17]$, which is valid in the double logarithmic limit, we end up with

$$
\frac{\partial^{2} x G_{A}\left(x, Q^{2}\right)}{\partial \ln (1 / x) \partial \ln \left(Q^{2} / \Lambda_{\mathrm{QCD}}^{2}\right)}=\frac{N_{c} C_{F} S_{\perp}}{\pi^{3}} Q^{2}\left[1-e^{-\left(2 \alpha_{s} \pi^{2} / N_{c} S_{\perp}\right) \frac{1}{Q^{2}} x G_{A}\left(x, Q^{2}\right)}\right],
$$


which exactly corresponds to the AGL equation (Eq. (10)). As demonstrated in Section 2 the GLR equation is a straightforward consequence from the AGL equation.

A last comment is important. Using the relation between $N$ and $x G$ from Ref. [17] a factor $e^{-\alpha_{s} \pi^{2} x_{01}^{2} x G_{A} / N_{c} S_{\perp}}$ remains in the right-hand side from Eq. (21). Therefore, the AGL equation only could be justified for a configuration of very small dipoles, as was discussed in [17]. Consequently, the connection between the dipole and AGL approaches deals with the correct physical relation between the function $N$ and the nuclear gluon distribution, given here by Eq. (17).

\section{Conclusions}

The AGL equation, obtained using the Glauber-Mueller approach, resums all multiple pomeron exchanges in the double logarithmic limit. Its solution allows us to make predictions which agree with the recent HERA data. In this paper we have analysed another equation which includes all multiple pomeron exchanges proposed by Kovchegov recently. The $\mathrm{K}$ equation was obtained in the leading logarithmic approximation using the dipole picture. We agree with this result. However, as the relation between the function $N$, whose evolution is described by the $\mathrm{K}$ equation, and the nuclear gluon distribution has some freedom, we obtained this connection considering the nuclear structure function in the Glauber-Mueller approach. Substituting this relation in the evolution equation for $N$ we have shown that it reduces directly to the AGL equation and, as a limit, to the GLR equation in the double logarithmic limit. This result shows that the AGL equation is a good candidate for the unitarized evolution equation at small $x$ in the DLA limit, supported by two different frameworks describing small $x$ phenomena.

Another candidate for the unitarized evolution equation was proposed by JalilianMarian et al. [28]. These authors have derived a general evolution equation for the gluon distribution in the limit of large parton densities and leading logarithmic approximation, considering a very large nucleus. This work is based on the effective Lagrangian formalism for the low $x$ DIS [29] and the Wilson renormalization group. In the general case the evolution equation is a very complicated equation, which does not allow one to obtain analytical solutions. Recently, these authors have considered the DLA limit on their result [30] and have shown that the evolution equation reduces to an equation with a functional form similar, but not identical, to the AGL equation. We believe that a more detailed analysis of the approximations used in both equations will allow one to demonstrate the equivalence of both equations in a common limit [31].

\section{Acknowledgements}

This work was partially financed by CNPq and by Programa de Apoio a Núcleos de Excelência (PRONEX), Brazil. 


\section{References}

[1] E.A. Kuraev, L.N. Lipatov and V.S. Fadin, Phys. Lett B 60 (1975) 50; Sov. Phys. JETP 44 (1976) 443; 45 (1977) 199;

Ya. Balitsky and L.N. Lipatov, Sov. J. Nucl. Phys. 28 (1978) 822.

[2] A.H. Mueller, Nucl. Phys. B 415 (1994) 373.

[3] A.H. Mueller and B. Patel, Nucl. Phys. B 425 (1994) 471.

[4] Z. Chen and A.H. Mueller, Nucl. Phys. B 451 (1995) 579.

[5] V.N. Gribov, E.M. Levin and M.G. Ryskin, Phys. Rep. 100 (1983) 1.

[6] J. Collins and J. Kwiecinski, Nucl. Phys. B 335 (1990) 89.

[7] J. Bartels, J. Blumlein and G.A. Schuler, Z. Phys. C 50 (1991) 91.

[8] J. Bartels and E.M. Levin, Nucl. Phys. B 387 (1992) 617.

[9] W. Zhu et al., Phys. Lett B 317 (1993) 200.

[10] E. Laenen and E.M. Levin, Nucl. Phys. B 451 (1995) 207.

[11] A.L. Ayala, M.B. Gay Ducati and E.M. Levin, Nucl. Phys. B 493 (1997) 305.

[12] A.L. Ayala, M.B. Gay Ducati and E.M. Levin, Nucl. Phys. B 511 (1998) 355.

[13] A.L. Ayala, M.B. Gay Ducati and E.M. Levin, Eur. Phys. J. C 8 (1999) 115.

[14] A.H. Mueller, Nucl. Phys. B 335 (1990) 115.

[15] A.H. Mueller, Nucl. Phys. B 437 (1995) 107.

[16] G.P. Salam, Nucl. Phys, B 461 (1996) 512.

[17] Y.U. Kovchegov, hep-ph/9901281.

[18] A.L. Ayala, M.B. Gay Ducati and E.M. Levin, Phys. Lett. B 388 (1996) 188.

[19] S.J. Brodsky and G.P. Lepage, Phys. Rev. D 22 (1980) 2157.

[20] Y.U. Kovchegov and A.H. Mueller, Nucl. Phys. B 529 (1998) 451.

[21] Yu. L. Dokshitzer, Sov. Phys. JETP 46 (1977) 641; G. Altarelli and G. Parisi, Nucl. Phys. B 126 (1977) 298;

V.N. Gribov and L.N. Lipatov, Sov. J. Nucl. Phys 15 (1972) 438.

[22] A.L. Ayala, M.B. Gay Ducati and V.P. Gonçalves, Phys. Rev. D 59 (1999) 054010.

[23] M.B. Gay Ducati and V.P. Gonçalves, SLAC-PUB-7968 (October 1998), hep-ph/9812459.

[24] V.N. Gribov, Sov. Phys. JETP 29 (1969) 483.

[25] N. Nikolaev and B.G. Zakharov, Z. Phys. C 49 (1990) 607 (1990);

S.J. Brodsky, A. Hebecker and E. Quack, Phys. Rev. D 55 (1997) 2584.

[26] W. Buchmuller, A. Hebecker and M.F. McDermott, Nucl. Phys. B 487(1997) 283.

[27] R.J. Glauber, Phys. Rev. 100 (1995) 242;

R.C. Arnold, Phys. Rev. 153 (1967) 1523;

T.T. Chou and C.N. Yang, Phys. Rev. 170 (1968) 1591.

[28] J. Jalilian-Marian et al., Phys. Rev. D 55 (1997) 5414; D 59 (1999) 014014; D 59 (1999) 014015.

[29] L. McLerran and R. Venugopalan, Phys. Rev. D 49 (1994) 2233.

[30] J. Jalilian-Marian et al., Phys. Rev. D 59 (1999) 034007.

[31] See, for example, E.M. Levin, hep-ph/9806434. 